\title{
EchoGéo
}

18 | 2011

Varia

\section{Pratique et enseignement de l'audiovisuel en géographie}

\section{Marie Chenet, Luisa Simoes et Quentin Laurent}

\section{OpenEdition}

1 Journals

Édition électronique

URL : https://journals.openedition.org/echogeo/12734

DOI : 10.4000/echogeo. 12734

ISSN : 1963-1197

Éditeur

Pôle de recherche pour l'organisation et la diffusion de l'information géographique (CNRS UMR 8586)

Référence électronique

Marie Chenet, Luisa Simoes et Quentin Laurent, « Pratique et enseignement de l'audiovisuel en géographie », EchoGéo [En ligne], 18 | 2011, mis en ligne le 05 décembre 2011, consulté le 31 juillet 2021. URL : http://journals.openedition.org/echogeo/12734 ; DOI : https://doi.org/10.4000/echogeo. 12734

Ce document a été généré automatiquement le 31 juillet 2021.

EchoGéo est mis à disposition selon les termes de la licence Creative Commons Attribution - Pas d'Utilisation Commerciale - Pas de Modification 4.0 International (CC BY-NC-ND) 


\title{
Pratique et enseignement de l'audiovisuel en géographie
}

\author{
Marie Chenet, Luisa Simoes et Quentin Laurent
}

\section{NOTE DE L'AUTEUR}

Les auteurs remercient les deux relecteurs dont les remarques pertinentes ont permis d'enrichir considérablement ce texte.

Les initiales MC, LS et QL correspondent aux initiales des auteurs qui ont rédigé les différentes parties.

1 Les géographes sont de plus en plus nombreux à réaliser des films documentaires et à les considérer comme de véritables documents de recherche. Les colloques sur le sujet se multiplient, notamment à l'étranger ${ }^{1}$, tandis que les doctorants incluent des films qu'ils ont tournés eux-mêmes en complément de leur mémoire de thèse ${ }^{2}$. A la suite de Xavier Browaeys (1999) et de Gaëlle Hallair (2007) notamment, il nous apparaît aujourd'hui opportun de revenir sur cette pratique de l'audiovisuel, notamment pour présenter l'enseignement qui est dispensé à l'UFR de Géographie de l'Université Paris 1 dès la Licence dans ce domaine. Pour cela, nous reviendrons dans un premier temps sur l'histoire des géographes qui ont eu recours tout au long du $20^{\mathrm{e}}$ siècle à l'usage de la caméra. Le lien entre l'outil cinématographique et la géographie n'est pas nouveau puisque les premiers films du géographe Jean Bruhnes ont été tournés en 1912. Même si ce dernier occupe alors une position marginale dans la géographie française, il n'en demeure pas moins le premier géographe à produire des films. Nous détaillerons ensuite l'apport de l'audiovisuel dans le champ de la recherche géographique, puisque l'image peut à la fois être analysée, produite et diffusée. Enfin, nous présenterons la façon dont les étudiants sont formés à l'Université Paris 1 aux techniques audiovisuelles dans le cadre de leur cursus de géographie. 


\section{Des géographes réalisateurs de films}

2 La réalisation de films documentaires n'est pas l'apanage des cinéastes professionnels puisque certains géographes ont eu très tôt recours à la caméra et au montage.

3 En 1912, le banquier et mécène Albert Kahn engage Jean Bruhnes comme directeur de la fondation des Archives de la planète. Ce fonds, qui possède plus de 100 heures de film ${ }^{3}$, témoigne de l'intérêt et de la contribution concrète d'un géographe français au cinéma. Jean Brunhes supervise de manière assez serrée la production photographique et cinématographique, comme en témoignent les instructions très précises qu'il donne à ses opérateurs4.

4 Mis à part quelques expériences isolées et pionnières (dont celle menée par Emmanuel de Martonne pendant les années 1930), il faut attendre les années 1950 pour voir des géographes au sein des institutions françaises se saisir à nouveau de la caméra. L'un des principaux points de production collective de films de géographes est sans nul doute le Centre Audiovisuel de l'ENS Saint-Cloud, inauguré en $1953^{5}$, mais que les géographes investissent réellement lors des années 1960. L'objectif initial est de produire une grande quantité de films à vocation pédagogique. Certains films de Jean-Louis Tissier, Philippe Pinchemel ou Jean-Pierre Deffontaines remplissent cependant d'autres fonctions et s'apparentent par bien des aspects à des films de recherche.

5 C'est à cette époque également qu'éclot une série d'expériences individuelles, souvent proches du film d'exploration : les géographes français commencent alors à réaliser des films liés à leur terrain de recherche. Les films de Claude Collin-Delavaud sont de bons exemples de ces premiers films connectés à des recherches universitaires. Claude Collin-Delavaud commence très tôt à filmer ses voyages. Au début des années 1950, avec l'appui institutionnel du Musée de l'Homme, il filme en Turquie Les nomades de Yörük Yayla. Il va ensuite parcourir le monde (Afghanistan, Pakistan, Chine, Vietnam, Mongolie, Brésil, Équateur, Chili). Sa méthode est celle du géographe explorateur dont la caméra l'accompagne à chaque voyage.

6 Les années 1960-1970 sont marquées, dans le domaine de l'audiovisuel, par d'importantes expérimentations cinématographiques (cinéma direct, documentaire subjectif, cinéma militant, etc.). A quelques exceptions près, il faut attendre les années 1980 pour que l'influence de ces nouveaux courants du cinéma documentaire ne se fasse sentir chez certains géographes, même si celle-ci ne s'est pas exercée de manière généralisée. On assiste plutôt à une multiplication des approches, et à une explosion de la production, imputable pour une bonne part aux progrès techniques. Avec l'avènement de la vidéo, les géographes disposent de caméras moins contraignantes en termes de poids et de technicité et moins onéreuses. Les outils de montage et de mixage sont eux aussi considérablement simplifiés. Tout cela permet au géographe d'acquérir une autonomie inédite et de ne plus être systématiquement obligé de se reposer sur une équipe de professionnels.

7 De toute évidence, et en dépit de certaines résistances rencontrées dans le monde universitaire où l'écrit demeure l'outil privilégié de production du savoir, on assiste depuis les années 1980 au développement timide d'une véritable géographie audiovisuelle, à l'image de ce qui se fait depuis un demi-siècle en anthropologie et en ethnologie ${ }^{6}$. Les géographes sont de plus en plus nombreux à réaliser des films ${ }^{7}$, et il est difficile de caractériser une production où les permanences sont aussi importantes que 
les évolutions. Pour schématiser, on peut dire que la production filmique actuelle des géographes se déploie entre deux extrêmes au milieu desquelles il existe un nuancier d'approches. D'une part, on trouve les films qui se rapprochent du reportage, où la voix-off savante donne la direction et le sens du film tout en s'appuyant sur des témoignages; l'image y est souvent utilisée comme illustration du propos exposé. A l'autre extrémité, on trouve des démarches qui tendent à réduire au maximum la posture du savant et donne une plus grande valeur aux effets de subjectivité ${ }^{8}$, par exemple en interrogeant le regard du chercheur. Entre ces deux postures, il existe une quantité d'approches variées, comme celles proposées par Xavier Browaeys (Université Paris 1 Panthéon-Sorbonne) ou Benoit Raoulx (Université de Caen Basse-Normandie). Le premier a œuvré pour la formation d'un grand nombre de géographes qui ont aujourd'hui des parcours très divers dans le domaine de la géographie ou dans le monde du cinéma. Sa production personnelle témoigne de cet éclectisme et il est difficile de lui attribuer un caractère d'ensemble ${ }^{9}$. Benoit Raoulx a acquis une formation de cinéaste au sein des célèbres Ateliers Varan. Ses films, très ancrés dans la géographie sociale, relèvent du « cinéma direct » en faisant de l'expérience du tournage le terrain même du géographe. (LS)

\section{Le cinéma comme démarche de recherche en géographie}

8 Le géographe peut travailler avec l'image animée et le son de deux manières : il peut les analyser ou les produire en vue d'une diffusion.

Un film, qu'il soit tourné par un cinéaste professionnel ou un géographe, peut être visionné et analysé afin de servir de document-source dans un travail de recherche géographique. Les images animées ont valeur d'archive qu'il s'agisse d'un film monté ou de rushes inutilisés mais souvent très intéressants. Ces documents peuvent alors faire l'objet d'une analyse comme n'importe quelle autre source de données géographiques. Mais ils peuvent également traduire à un moment donné la vision que le réalisateur porte sur un territoire ou un processus. Tout cinéaste qui filme propose un regard, que cela soit à travers sa manière de filmer ou la structure plan à plan de son récit. Le géographe doit décoder le langage cinématographique pour faire émerger cette vision. Il s'agit alors de décortiquer plan par plan le film, de comprendre les choix d'images (choix de cadre, d'angles de vue, d'échelle de plans, etc.) mais aussi de mixage (bande sonore, éventuelle voix off, interviews, etc.) et de montage (les grandes parties et les jointures du récit). Le film, là encore, acquiert le statut de document analysé par le géographe.

10 Le géographe peut également lui-même réaliser des films ${ }^{10}$. Avec la simplification de la technique, il peut aujourd'hui facilement se former aux différentes étapes de la réalisation et produire des œuvres documentaires. L'enjeu de la production de film dans une démarche de recherche est alors d'assembler des images et des sons qui ne vont pas servir d'illustrations mais bien de base à l'élaboration d'un propos, d'un point de vue. Par ce procédé, le géographe doit se plier à une nouvelle forme de réflexion, puisque l'on ne présente pas de la même façon un raisonnement et des résultats par écrit et par vidéo. Le recours à l'audiovisuel impose de construire une trame narrative, de trouver des liens de causalité spécifiques aux images et aux sons. En s'emparant des outils du cinéma, le géographe se donne donc pour objectif de donner à voir et à 
entendre le monde en établissant des rapports nouveaux afin de construire un raisonnement et de proposer une thèse d'interprétation du monde. Cette vision rejoint celle proposée par Jean Vigo qui, en 1930, lors de la projection de son très beau A propos de Nice, définissait le film documentaire comme un « point de vue documenté »11.

11 Pour aboutir à ce résultat, le géographe cinéaste doit se former aux trois étapes nécessaires à l'élaboration d'un film : l'écriture, le tournage et le montage. La première étape, la phase d'écriture, est très similaire au travail préparatoire du géographe avant de partir sur son terrain. Le cinéaste, comme le géographe, se documente et tente d'anticiper le réel afin d'établir des hypothèses qui contribuent à l'élaboration d'une thèse qui sera confirmée ou infirmée par l'investigation sur le terrain (lors du tournage). C'est donc une étape relativement commune au travail de recherche universitaire, qui aboutit à l'écriture d'un synopsis et d'un scénario provisoire.

Passée cette phase d'écriture, le géographe dispose de deux outils spécifiques au cinéma : le tournage et le montage. Tourner des plans, c'est donner à voir et faire entendre un paysage, une scène, un objet à un moment précis. La façon dont la scène est filmée traduit le regard du réalisateur, son interprétation. Idéalement, le propos autrement appelé le fond - devrait toujours être connecté à la forme. Pour cela, le géographe doit acquérir une maîtrise du langage et des techniques cinématographiques qui, par le choix du cadre, de l'angle de prise de vue, de la lumière ou du son permettent de donner du sens à l'image.

Le géographe dispose également du montage pour construire son raisonnement. Cette étape consiste à assembler les images et les sons de façon à former un tout cohérent. C'est le moment de définir la manière dont le propos va être présenté, de choisir les scènes et les personnages qui appuieront la démonstration, mais également de jouer sur des oppositions de plans, de confronter des discours. Pour le géographe habitué à l'écrit, il faut alors être très vigilant: la tentation est grande de reproduire les formes narratives communes aux discours universitaires (introduction, développement en trois parties et conclusion). L'enjeu ici est d'adopter une forme cinématographique et donc de raconter une histoire. On le voit donc, pour qu'un film soit réussi, pour que le propos soutenu soit intelligible et engage une réflexion, il importe au géographe cinéaste de travailler à la fois sur le fond et sur la forme du film qu'il réalise. Bien souvent, nous avons pu constater que cette forme était négligée par les apprentis cinéastes, en raison d'un manque de culture cinématographique.

La réalisation d'un film documentaire prend tout son sens au moment de la projection. Plus que le texte, le film permet le débat. Le spectateur qui visionne le film est dans le domaine de l'émotionnel, et cette subjectivité lui donne envie de réagir. De plus, le visionnage est, dans une salle de cinéma, une expérience collective qui permet la discussion. Le réalisateur peut alors confronter son point de vue à celui du public, qu'il soit spécialiste ou non, mais aussi recueillir la réaction des personnages filmés. Ainsi, dans Chronique d'un été ${ }^{12}$, les ethnologues Jean Rouch et Edgar Morin, après avoir projeté la première version de leur œuvre, filment la salle de cinéma où sont réunis les protagonistes du film et enregistrent leurs réactions. Dans une dernière étape, ils se filment eux-mêmes déambulant dans le Musée de l'homme et analysant ce qu'ils tirent de leur expérience. La vie d'un film est donc sans fin puisque la réaction des spectateurs peut elle-même constituer une part du film. Et c'est là encore une des forces de l'image animée, puisque plusieurs publics, d'origines différentes, peuvent être touchés en même temps et de façon différente. 
15 En utilisant l'audiovisuel, le géographe dispose donc d'une méthode alternative pour tenter d'observer et d'expliquer le monde. Et c'est cette alternative à l'écrit qui est généralement appréciée des étudiants en géographie qui sont initiés à la réalisation de films documentaires. (MC et QL)

\section{Enseigner l'audiovisuel aux étudiants géographes}

La maîtrise de l'audiovisuel constitue un atout déterminant pour l'enrichissement du discours géographique. C'est pour cette raison que les géographes Xavier Browaeys et Paul Chatelain ont créé, au milieu des années 1990, une formation à la réalisation de films documentaires au sein de l'UFR de Géographie de l'université Paris I. Dès la deuxième année de Licence et jusqu'en Master, les étudiants sont formés à l'analyse de films et à la réalisation.

En Licence, les étudiants réalisent par groupe de 4 ou 5 un court-métrage de 5 minutes environ sur des thématiques géographiques (cette année, la place parisienne, par exemple). Pendant un semestre, ils sont initiés aux différentes étapes de la réalisation de films : l'écriture, le tournage, le montage et le mixage. Le matériel de tournage (caméras, pieds, micros) comme celui de montage (utilisation du logiciel Casablanca) leur sont fournis par l'UFR. La formation est complétée par le visionnage de films dont le propos et la structure sont analysés. Ainsi, environ 6 films sont réalisés chaque semestre par une trentaine d'étudiants.

En Master, les étudiants doivent réaliser un moyen-métrage (20 à 40 minutes selon le sujet et le terrain) qu'ils présentent en fin d'année, accompagné d'un complément écrit. Ils sont encadrés à la fois par un enseignant spécialiste de la thématique traitée et par un géographe formé à la vidéo. Le film est réalisé en autonomie, depuis l'écriture jusqu'au montage final. Pour cela, les étudiants disposent d'une formation d'une dizaine de séances au premier semestre qui reposent sur deux fondements : d'une part la connaissance de l'histoire du cinéma documentaire et des grands classiques du genre, d'autre part la pratique du tournage. Ainsi, les étudiants réalisent chaque semaine des exercices de tournage pour approfondir leur maîtrise de la technique (filmer un lieu, un geste, un portrait, etc.). Pour cela, la technique du "tourné-monté" est adoptée. Cette méthode consiste à tourner les plans dans l'ordre chronologique de visionnage et en une seule prise, sans recourir au montage. Elle impose à l'étudiant de penser le déroulé de son film avant le tournage, de sélectionner au moment du repérage les étapes à filmer et de se projeter dans la forme finale de son film sans se reposer sur le «confort " du montage ${ }^{13}$. Après cette phase de formation, les étudiants partent seuls sur leur terrain, en France ou à l'étranger, pour des durées de un à cinq mois. Le montage a ensuite lieu à Paris, à l'Université. Là encore, le matériel est fourni par l'UFR. Certains des films réalisés par les étudiants de Master sont visibles sur le site Doc2Géo ${ }^{14}$ ou projetés lors du festival Territoires en Images organisé par l'association Arrimage à l'Institut de Géographie de Paris. (MC et QL) 


\section{BIBLIOGRAPHIE}

Browaeys X., 1999. Géographie, image et vidéo. Pour une pratique de l'audiovisuel.

L'Information géographique, vol 63, p. 25-32.

Calberrac Y., 2010. Terrains de géographes, géographes de terrain. Communauté et imaginaire disciplinaires au miroir des pratiques de terrain des géographes français du XXe siècle. Thèse de doctorat en géographie, Université Lumière Lyon 2.2 volumes ( 392 p et 400 p) et un film documentaire Ce qui fait terrain - Fragments de recherches (52 minutes).

Buire C., 2011. À travers pratiques citadines et tactiques citoyennes, la production du droit à la ville au Cap (Afrique du Sud). Thèse de doctorat en géographie, Université Paris X-Nanterre. 1 volume (411 p.) et 1 DVD.

Delamarre M., Bonhomme M., 1993. La méthode des missions des Archives de la Planète. In Autour du monde: Jean Brunhes, regards d'un géographe, regards de la géographie. Boulogne,Musée Albert Kahn, p. 194 sq.

Dubost M., 2004. Le centre audiovisuel de Saint-Cloud et ses origines. In Pastre-Robert B. (dir.), Cinéma pédagogique et scientifique : à la redécouverte des archives, Lyon, ENS.

Garrett B., 2001. Videographic geographies: Using digital viedo for geographic research. Progress in Human Geography, vol. 35.

Hallair G., 2007. Vidéo et pratique de la Géographie. EchoGéo [En ligne], º 2. URL : http:// echogeo.revues.org/1643

Lefort I. et Calberac Y., 2009. Faire d'un fonds documentaire un patrimoine. La valorisation des films pédagogiques produits par l'Ecole normale supérieure de Saint-Cloud. In Hiraux, F. (dir.), Les archives audiovisuelles. Politiques et pratiques dans la société de l'information, Bruxelles: AcademiaBruylant.

Pink S., 2007. Doing Visual Ethnography. Londres, Sage.

\section{NOTES}

1. Citons entre autres la conférence annuelle de la Royal Geographical Society qui, en août 2011 , ne consacraient pas moins de trois sessions traitant de géographie et de vidéo (Projects, participants and perspectives - participatory video in practice; Spaces, places and communities, picturing places through film and video ; Sensory video and the embodied spaces of film and video).

2. En ce sens, deux thèses de doctorat peuvent être citées : celle de Yann Calbérac (2010) et celle de Chloé Buire (2011)

3. Des extraits de ces archives sont visibles sur le site du musée Albert Kahn: http://albertkahn.hauts-de-seine.net/archives-de-la-planete/

4. Toutes les instructions de J. Brunhes sont explicitées en détail dans l'article de Mariel JeanBrunhes Delamarre et Marie Bonhomme (1993).

5. Voir Dubost (2004) et Lefort et Calberac (2009).

6. Bradley Garrett soulève la question de savoir pourquoi l'audiovisuel n'a pas fait l'objet d'un engouement aussi considérable chez les géographes qu'en anthropologie (Garrett, 2011). 
7. A l'Université Paris 1 , on peut citer les noms de Béatrice Collignon, Martine Tabeaud ou Jean Marie Théodat.

8. Ici, l'anthropologie audiovisuelle constitue une référence majeure (Pink, 2007), souvent citée par certains géographes.

9. Voir par exemple Mémoire d'un lieu : la Bibliothèque de l'Institut de géographie de Paris (1999, 20'), Karakorum (2003, 26'), ou La ZAC aux folles (co-réal. M. Tabeaud, 2003, 14') sur le site http:// sites.google.com/site/doc2geo/home .

10. En ce sens, la thèse de doctorat de Chloé Buire (2011) sur la production du droit à la ville au Cap, en Afrique du Sud est tout à fait originale. C. Buire a tourné des petites séquences indépendantes les unes des autres, ce qui lui permet de s'interroger à la fois sur la manière de filmer et sur ce que révèlent les images.

11. Texte prononcé par Jean Vigo au Vieux-Colombier, le 14 juin 1930, lors de la seconde projection de son film A propos de Nice.

12. J. Rouch et $\mathrm{E}$. Morin, Chronique d'un été, 1961 (90 mn). Disponible en DVD, Arte vidéo.

13. Le montage permet de sélectionner des plans tournés, d'ajuster leur durée et de les agencer dans l'ordre que l'on désire. Ne pas recourir au montage, c'est s'obliger à ne filmer que ce qui est utile, d'estimer la durée idéale des plans et de tourner dans l'ordre chronologique les différentes étapes du film.

14. Doc2géo: site internet regroupant de nombreux films de géographes http:// sites.google.com/site/doc2geo/. Parmi les films réalisés par des étudiants de Master, citons notamment : Du passé faisons table rase... Pékin 2003 de G. Lavoué (2003), La culture du risque de F. Flohic et F. Geyer (2003), Misti es-tu là ? de L. Aïssani et C. Kopp (2006), Rive Gauche, Paris d'A. Dionnet (2006), Dakar Terminus de Q. Laurent (2008), 4 hommes et 1 jour à Placodji de L. David (2009).

\section{RÉSUMÉS}

Les géographes ont depuis le début du $20^{\mathrm{ème}}$ siècle recours à l'usage de l'audiovisuel, qu'il s'agisse d'analyser ou de produire des films dans le cadre de recherches. Ils ont donc dû se former aux outils et aux méthodes de l'image animée et du son. L'article propose ainsi de revenir sur l'histoire des géographes qui ont utilisé la vidéo dans le cadre de leur recherche avant de présenter l'apport de l'audiovisuel en géographie. Il termine sur une présentation de la formation proposée par l'Université Paris 1 aux étudiants de géographie dans ce domaine.

Since the beginning of the 20th Century, the geographers use audiovisual methods, through frame analysis or film direction as part of their researches. Therefore, they had to train in audiovisual tools and methods. The paper presents first a short history of geographers who directed films. Then the contribution of audiovisual in geographical researches is treated. Lastly, the formation in video offered to the geography student in the University Paris 1 is presented. 
INDEX

Keywords : audiovisual formation, direction, documentary film, frame analysis, geography

Mots-clés : analyse d'images, film documentaire, formation audiovisuelle, géographie, réalisation

\section{AUTEURS}

\section{MARIE CHENET}

Marie Chenet, Marie.Chenet@cnrs-bellevue.fr, est maître de conférences à l'Université Paris 1, responsable de l'atelier Géovidéo de l'UFR de Géographie de l'Université Paris 1, et membre du Laboratoire de Géographie Physique (CNRS-UMR 8591).

\section{LUISA SIMOES}

Luisa Simoes, luisa.gomes-simoes@univ-paris1.fr, est doctorante contractuelle et chargée de cours, Université Paris 1, membre de Géographie-cités (CNRS-UMR 8504) et chercheuse associée à la Bibliothèque Nationale de France, département audiovisuel.

\section{QUENTIN LAURENT}

Quentin Laurent, quentinlaurentpro@gmail.com, est assistant de production aux Productions de l'œil sauvage, ancien étudiant de géographie à l'Université Paris 1, titulaire d'un M2 Pays Emergents et en Développement. 